\title{
Adipogenic niches for melanoma cell colonization and growth in bone marrow
}

\author{
Juan Wang ${ }^{1,2,8}$, Guang-liang Chen ${ }^{3,4,8}$, Shan Cao ${ }^{2,8}$, Ming-chun Zhao ${ }^{5}$, Yong-qing Liu' ${ }^{6}$ Xiao-Xiang Chen ${ }^{2}$ and \\ Cheng Qian ${ }^{7}$
}

Bone marrow (BM) adipocytes are abundant in BM and may be involved in the process of bone metastasis. However, their behaviors in metastatic BM niches during bone metastasis have not been fully explored. In this study, intracardiac transplantation of B16-F10 melanoma cells into immunocompetent C57BL/6 mice was performed. Tibial marrow sections were stained with hematoxylin and eosin, Masson's trichrome, tartrate-resistant acid phosphatase, and fatty acid-binding protein 4 (FABP4) and analyzed using a histomorphometric system. The results showed that the number of BM adipocytes rapidly increased in melanoma metastatic $B M$ niches, which were in direct contact with metastasizing melanoma cells and acted as a tumor stromal population in the BM-melanoma niche. Melanoma cell-derived factors could enhance BM adipogenesis, which promotes melanoma cell proliferation and cell cycle transitions. Moreover, BM adipocytes might aid in the modification of the osteolytic BM microenvironment. These results indicate that an increase in the number of BM adipocytes in a metastatic BM niche may facilitate melanoma cell colonization and growth in BM. BM adipocytes might therefore support the development of bone metastases.

Laboratory Investigation (2017) 97, 737-745; doi:10.1038/labinvest.2017.14; published online 20 February 2017

Bone metastasis is a detrimental complication in cancers, including malignant melanoma (MM). ${ }^{1,2} \mathrm{MM}$ patients with bone metastases display predominantly osteolytic lesions in their bones, ${ }^{3}$ and these are mainly caused by aberrant activated bone-absorbing osteoclasts. Although osteoclasts are the major cellular component during bone destruction and interactions with tumor cells, there is no evidence supporting the notion that osteoclasts provide niches for cancer cell colonization during bone metastasis. Recent studies have revealed the presence of osteogenic niches in prostate cancer, ${ }^{4}$ acute myelogenous leukemia (AML), ${ }^{5}$ and breast cancer. ${ }^{6}$ However, the characteristics of the niche in which melanoma cell colonization and growth occur in bone marrow (BM) have not been described.

$\mathrm{BM}$ adipocytes, which are one of most abundant stromal cell types in BM, are present in both white and brown adipose tissue. ${ }^{7}$ The number of BM adipocytes increases with age ${ }^{8}$ and obesity. ${ }^{9}$ A higher incidence of bone metastasis has been observed in elderly patients than in younger patients, ${ }^{10}$ and this might indicate a role for $\mathrm{BM}$ adipocytes in bone metastasis. In recent studies, BM adipocytes that were induced by obesity were shown to promote cancer cell growth in the bone-tumor niche ${ }^{11,12}$ and to enhance osteoclastogenesis via chemokine (C-X-C motif) ligand-1/-2 (CXCL-1/-2) and osteopontin (OPN). ${ }^{11,13}$ In addition, adipocytes that synthesize cytokine-like proteins called adipokines, which include leptin, interleukin 6 (IL-6), insulin-like growth factor 1 (IGF-1), and CXCL-1/-2, are also potentially involved in metastasis. ${ }^{14}$ Furthermore, BM adipocytes negatively regulate the hematopoietic stem cell (HSC) pool, ${ }^{15,16}$ which is shared with metastasizing cancer cells. ${ }^{4,17}$ This regulation may affect the BM metastatic niche. However, whether BM adipocytes influence the metastatic niche during osteolytic metastases remains unknown.

In the present study, we used a well-established bone metastasis mouse model to show that the numbers of unilocular and multilocular BM adipocytes are rapidly increased in the BM metastatic niche. Importantly, melanoma

\footnotetext{
Department of Internal Medicine, Shanghai First People's Hospital, Affiliated to Shanghai Jiao Tong University, Shanghai, China; ${ }^{2}$ Department of Rheumatology, Renji Hospital, Affiliated to Shanghai Jiao Tong University, School of Medicine, Shanghai, China; ${ }^{3}$ Department of Internal Medicine 3, University of Erlangen-Nuremberg, Erlangen, Germany; ${ }^{4}$ Department of Oncology, Minhang Hospital, Fudan University, Shanghai, China; ${ }^{5}$ Department of Pathology, Guilin People's Hospital, Guilin, China; ${ }^{6}$ Department of Pathology, Guigang City hospital of Traditional Chinese Medicine, Guigang, China and 'Department of Thoracic Surgery, Zhongshan hospital, Fudan University, Shanghai, China

Correspondence: Dr Cheng Qian, MD, Department of Thoracic Surgery, Zhongshan hospital, Fudan University, No.180, Xuhui District, Fenglin Road, Shanghai 200032, China; E-mail: qiancheng.zs@foxmail.com

${ }^{8}$ These authors contributed equally to this work.

Received 22 June 2016; revised 19 January 2017; accepted 20 January 2017
} 
cells are directly in contact with BM adipocytes, which constitute an additional stromal cell population in the tumor area during melanoma progression. Moreover, dual effects of melanoma cells on BM adipocytes were observed. These findings provide evidence supporting the importance of BM adipocytes in bone metastasis.

\section{MATERIALS AND METHODS Mice Treatment}

Male C57BL/6 mice (10 weeks old) were purchased from SLAC (Shanghai Laboratory Animal, Shanghai) and maintained at $25^{\circ} \mathrm{C}$ with a 12-hour light/dark cycle. The procedure used for the intracardiac (i.c.) injections was previously described by Bakewell et al..$^{18}$ and Campbell et al. ${ }^{19}$ Briefly, the mice were anesthetized using isoflurane (RWD Life Science, San Diego, CA, USA). A twenty-nine-gauge needle (Micro-Fine, BD) was used to inject either $1 \times 10^{5}$ B16-F10 cells in $100 \mu \mathrm{l}$ of PBS or only PBS (vehicle) into the left cardiac ventricle of each mouse. The mice were monitored daily for up to 14 days after injection and killed at the indicated time points (Figure 1a). Mice with visible extrapleural intrathoracic tumors were excluded from the analysis. All of the animal experiments were approved by the Animal Care and Use Committee at Shanghai Renji Hospital and Shanghai First People's Hospital, which is affiliated with Shanghai Jiao Tong University.

\section{Cell Cultures}

The murine melanoma cell line (B16-F10) was purchased from the Type Culture Collection of the Chinese Academy of Sciences, Shanghai, China. Cells were maintained in DMEM (Invitrogen, Thermo Fisher Scientific) containing $100 \mathrm{ml} / \mathrm{l}$ fetal bovine serum (FBS), $100 \mathrm{IU} / \mathrm{ml}$ penicillin, and $10 \mu \mathrm{g} / \mathrm{ml}$ streptomycin (Invitrogen, Thermo Fisher Scientific) at $37^{\circ} \mathrm{C}$ (5\% $\mathrm{CO}_{2}, 95 \%$ air). The B16-F10 cells were transduced with pGLV3-H1-GFP-Puro plasmids (Biogot Technology) using Lipofectamine 3000 (Invitrogen, USA). Stable green fluorescent protein (GFP)-expressing B16-F10 cells were selected using complete DMEM medium containing $1.5 \mu \mathrm{g} / \mathrm{ml}$ puromycin (InvivoGen, USA) for 7-10 days.

\section{Adipocyte Differentiation Assay}

Mouse BM mesenchymal stem cells (BM-MSC) were prepared as previously described. ${ }^{11,20} \mathrm{BM}-\mathrm{MSC}$ or the murine adipocyte cell line (14F1.1 cells, kindly provided by Prof. Dov Zipori of the Weizmann Institute of Science, Rehovot, Israel) were differentiated into $\mathrm{BM}$ adipocytes as previously reported. ${ }^{11}$ As shown in Figure 2a, BM-MSC cells or 14F1.1 cells were pre-treated with adipogenic cocktail (adipo-coc: $5 \mu \mathrm{g} / \mathrm{ml}$ insulin, $0.5 \mathrm{mM}$ 3-isobutyl-1-methylxanthine, $1 \mu \mathrm{M}$ dexamethasone) for 2 days. Then, the tumor-conditioned medium (TCM) was collected from the B16-F10 cells, which were cultured in FBS-free medium for $24-48 \mathrm{~h}$ and mixed with adipo-coc $(40 \%)$ as the medium used for the adipocyte differentiation assay. Adipocytes were harvested for gene expression profiling or Oil Red O staining.

\section{Adipocytes and B16 Cells Co-culture}

The 14F1.1 cells were differentiated into mature BM adipocytes in 24-well plates as described above. B16-F10 cells $\left(1 \times 10^{3} / 10^{5} / 10^{6}\right)$ were placed in $0.4-\mu \mathrm{m}$ pore inserts (Milipore) for co-culture experiments (Figure 3a). After $48 \mathrm{~h}$ of incubation, the adipocytes were harvested for gene expression profiling or staining.

\section{Proliferation Assay and Cell Cycle Analysis}

Mature BM adipocytes were cultured overnight in DMEM medium containing 2\% FBS and harvested as the adipocyteconditioned medium (Ad-CM). The WST-1 cell proliferation and cytotoxicity assay kit (Beyotime Institute of Biotechnology, China) was used for detecting proliferation in B16-F10 cells after incubation in Ad-CM or control DMEM medium according to the manufacturer's instructions. For cell cycle analysis in flow cytometry, B16F10 cells were treated with control medium ( $0 \%$ Ad-CM), $50 \%$ Ad-CM, or $100 \%$ Ad-CM for $48 \mathrm{~h}$.

\section{Bone Histology and Histomorphometry}

Mouse femurs and tibias were fixed in $4 \%$ formalin overnight and decalcified in 14\% EDTA for 2-3 weeks. Long bones were embedded in paraffin and sliced into equivalent coronal sections through the center of the bone at a thickness of $2 \mu \mathrm{m}$. Histological sections were stained with HE, Masson's trichrome, and tartrate-resistant acid phosphatase (TRAP) (\#387, Sigma-Aldrich, St. Louis, MO, USA) according to the manufacturer's instructions. Osteoclast number and size and trabecular bone area were measured as described in our previous report. ${ }^{11}$ For immunostaining, de-paraffinized, ethanol-rehydrated tissue sections were labeled with the following antibodies: anti-fatty acid-binding protein 4 (FABP4) antibodies (ab92501; Abcam; dilution 1:500) or anti-GFP antibodies (ab13970; Abcam; dilution 1:1000). The section preparation and labeling were performed by technicians from Shanghai Zuo Cheng Bio.

\section{Quantitative RT-PCR}

Cell lysates were homogenized using TRIzol reagent (Invitrogen), and RNA was then isolated according to the manufacturer's instructions. RNA was reverse-transcribed into cDNA using a PrimeScript RT reagent kit with gDNA Eraser (Takara Biotechnology (Dalian)). Quantitative real-time PCR was performed using SYBR Premix Ex Taq II (Tli RNase H Plus) on an Applied Biosystems 7900HT Fast Real-Time PCR System. Samples were analyzed in duplicate. $\beta$-actin was used to normalize the RNA content of the samples. The primer sequences are listed in Supplementary Table 1.

\section{Enzyme-Linked Immunosorbent Assay (ELISA)}

Leptin levels in serum were measured using ELISA kits (R\&D Systems) according to the manufacturer's instructions. 
a

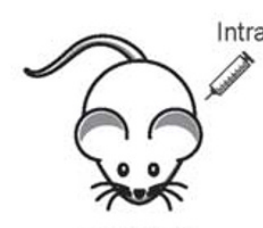

C57BL/6

12 week-old

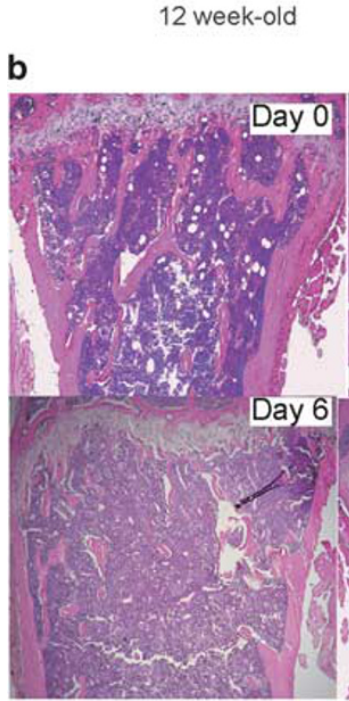

d
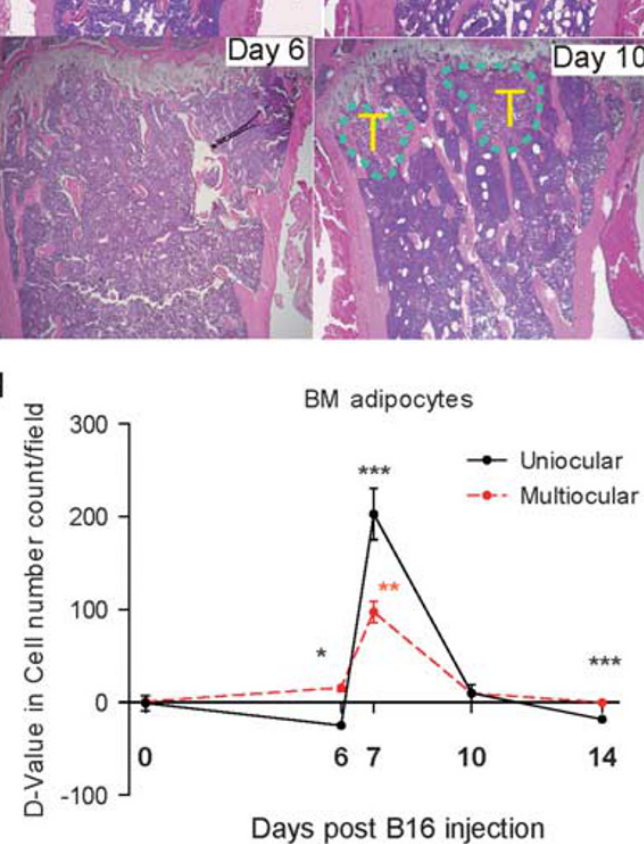

C

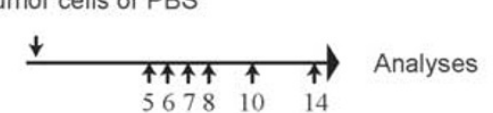

Days after injection
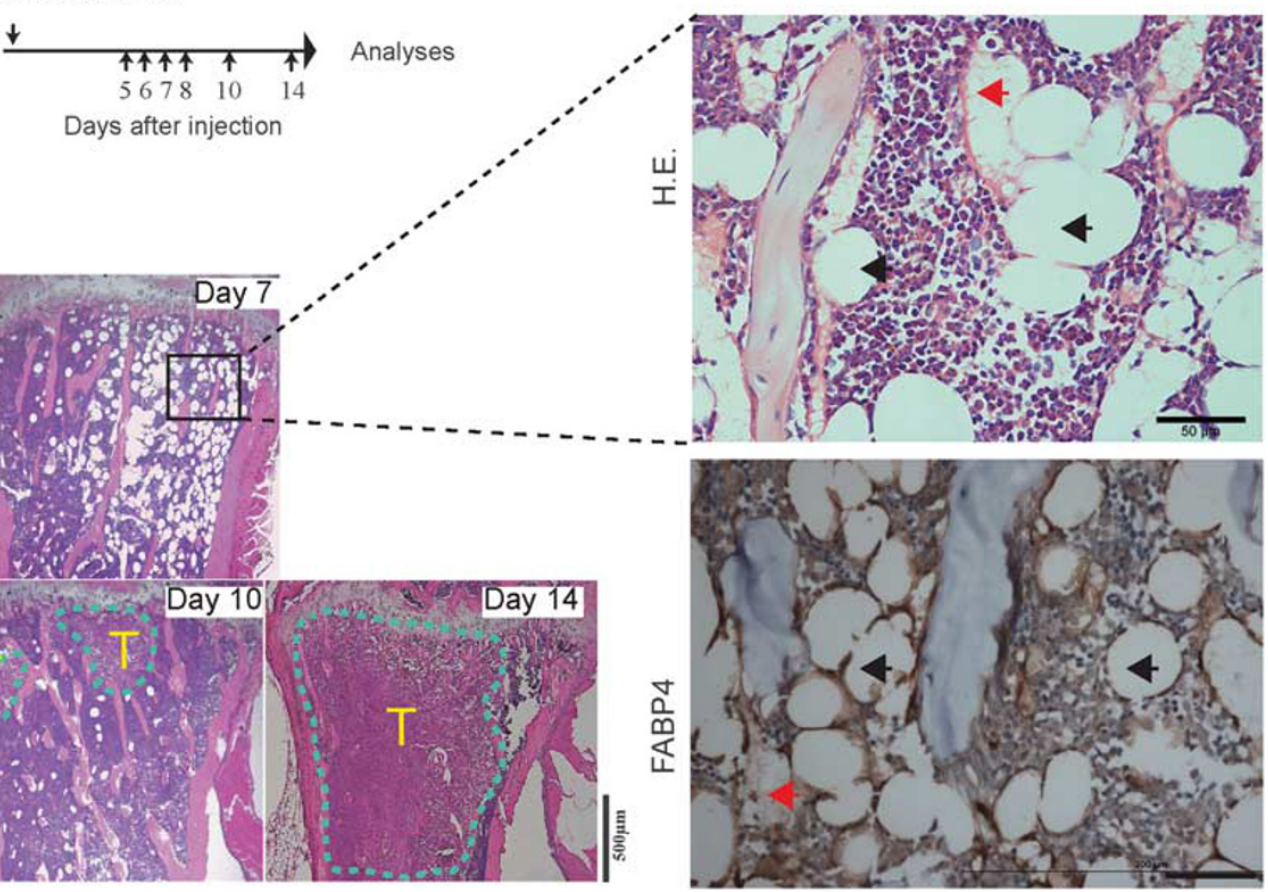

Day 14
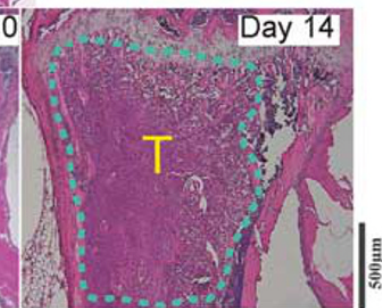

e

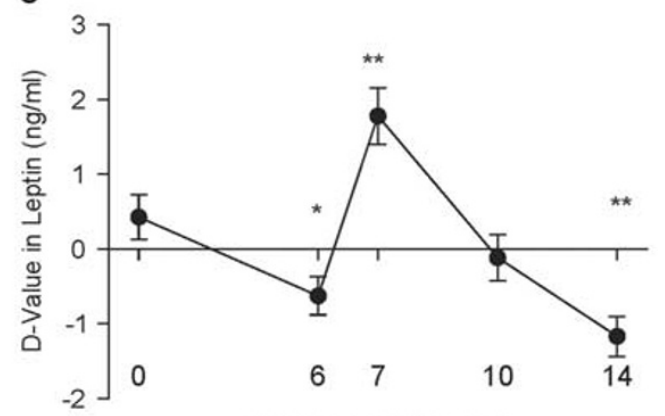

Days post B16 injection

Figure 1 A significant increase was observed in the number of BM adipocytes in the metastatic bone niche. (a) Experimental scheme: B16-F10 cells $\left(1 \times 10^{5}\right)$ were injected (intracardiac, i.c.) into 10-12-week-old immunocompetent C57BL/6 mice. The mice were killed for analysis at the indicated time points (days) post-tumor inoculation. (b-c) HE-stained slides of tibial tissues (scale bar, $500 \mu \mathrm{m}$ ). Unilocular and multilocular BM adipocytes are indicated by black and red arrows, respectively (scale bar, $50 \mu \mathrm{m}$ ). FABP4 staining was performed on tibial bone sections obtained on day 7 . Tumor area is marked with a yellow ' $T$ '. (d) The difference values (D-values) in the numbers of BM adipocytes of tibial tissues between tumor-injected and vehicleinjected mice were calculated. (e) The D-values in serum levels of leptin $(\mathrm{ng} / \mathrm{ml})$ between tumor-injected mice and vehicle-injected mice were analyzed. All of the statistical data are shown as means \pm s.e.m.; $n=5-6$ per group. ${ }^{*} P<0.05$, ${ }^{*} P<0.01,{ }^{* * *} P<0.001$. BM, bone marrow; $\mathrm{HE}$, hematoxylin.

\section{Statistics}

All data are presented as the mean \pm s.e.m. Statistical significance was determined using unpaired Student's $t$ tests implemented using GraphPad Prism software $\left({ }^{\star} P<0.05\right.$, $\left.{ }^{* *} P<0.01,{ }^{* * *} P<0.001\right)$.

\section{RESULTS}

\section{The number of BM Adipocytes is Increased in the BM Metastatic Niche}

To reveal how the cellular components of the BM metastatic niche change during bone metastasis, immunocompetent
C57BL/6 male mice (10-12 weeks old) were inoculated via i.c. injection and then monitored at previously determined time points (Figure 1a). In the tibial BM HE-stained sections, increased numbers of unilocular and multilocular BM adipocytes were observed in the tumor cell-inoculated mice on day 7 post-tumor transplantation compared with the PBSinjected mice (Figures 1b-d). However, a dramatic decrease in the numbers of unilocular BM adipocytes was observed on day 14 post-melanoma cell injection compared with the control group (Figures $\mathrm{lb}$ and $\mathrm{d}$ ). Immunohistochemical staining for FABP4, a specific marker of adipocytes, 
a

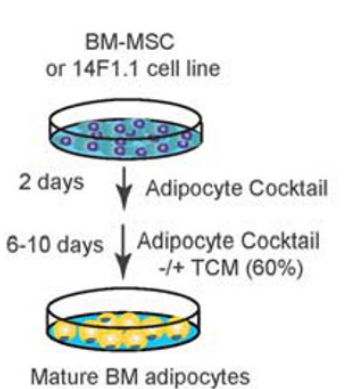

b

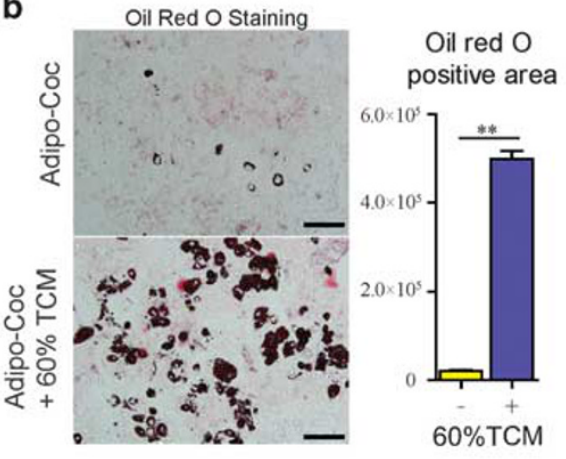

c

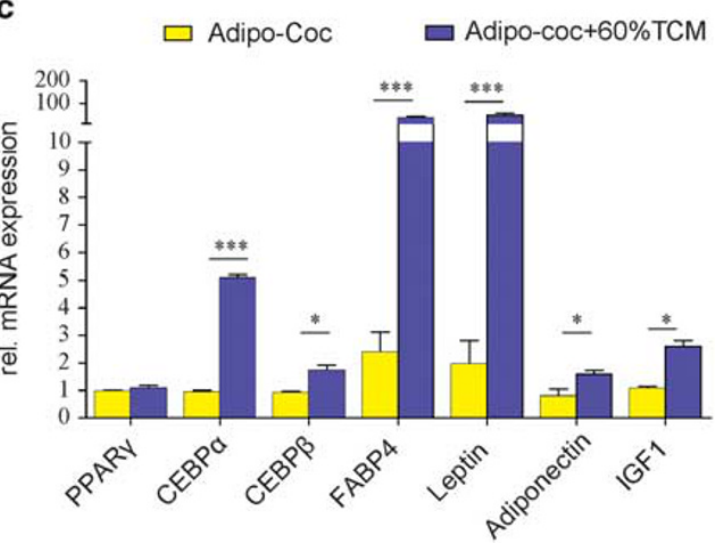

Figure 2 Tumor-derived factors promote BM adipocyte differentiation.(a) Experimental setting: isolated BM MSC or 14F1.1 cells (a murine BM adipogenic cell line) were seeded on cell plates and differentiated into adipocytes by the addition of adipogenic cocktail (adipo-coc) for 2 days with/ without a 2:3 mixture of the conditioned medium obtained from B16F10 cells (TCM) for an additional 6-10 days. (b) Representative photographs of Oil Red $\mathrm{O}$ staining of adipocyte cultures in 24-well plates. A representative image of at least 3 independent experiments carried out in triplicate is shown. Quantification of the intensity of staining was performed using ImageJ. (c) Expression of adipocyte marker genes in cell cultures. All of the statistical data are presented as means \pm s.e.m. of three independent experiments carried out in triplicate. ${ }^{*} P<0.05,{ }^{* *} P<0.01,{ }^{* *} P<0.001$. BM, bone marrow; MSC, mesenchymal stem cells; TCM, tumor-conditioned medium.

confirmed that the stained cells were adipocytes (Figure 1c). Furthermore, serum levels of leptin, which is secreted mainly by adipocytes, ${ }^{21}$ were also detected and found to change in parallel with the observed changes in cell numbers of BM adipocytes (Figure 1e). Taken together, these data suggest that $\mathrm{BM}$ adipocytes might be active players in the development of melanoma bone metastases.

\section{Dual Effects of Melanoma Cells on BM Adipocytes: Pro-differentiation and De-differentiation}

To test whether the tumor cells could promote bone marrow adipocyte differentiation, isolated BM-MSC or 14F1.1 cells were differentiated into mature adipocytes using an adipogenic cocktail with or without the addition of $60 \%$ TCM (Figure 2a). We observed that TCM was able to promote the differentiation of adipocytes, which was confirmed by Oil Red $\mathrm{O}$ staining and profiling of adipocyte marker genes (Figures $2 \mathrm{~b}$ and $\mathrm{c}$ ). However, previous studies have shown that adipocytes can also undergo a de-differentiation process when co-cultured with tumor cells. ${ }^{22-24}$ To address whether the process was dependent on the number of tumor cells, a transwell co-culture system was used, ie, a range of melanoma cell densities (from $10^{3}$ to $10^{6}$ cells/well) were co-cultured with mature adipocytes (Figure $3 a$ ). As shown in Figure $3 b$, delipidation of mature adipocytes were induced to a greater extent at the highest density of melanoma cells $\left(10^{6}\right.$ cells/well $)$ compared with at lower densities of melanoma cells $\left(10^{3}\right.$ or $10^{5}$ cells/well). Consistent with these findings, the expression of genetic markers of adipocytes, such as CCAAT/enhancer binding protein beta $(C E B P \beta)$, peroxisome proliferator activated receptor gamma (PPAR $\gamma)$, FABP4 and Leptin, were decreased while delta like non-canonical Notch ligand 1 (Pref- ${ }^{1} I L-1 \beta$, IL-6, and C-C Motif chemokine ligand 2 (MCP-1) were enhanced at the highest density of melanoma cells (Figure 3d).

\section{BM Adipocyte Provide a Niche for Melanoma Cells Colonization}

BM adipocytes might support the development of tumor cell bone metastasis. To examine whether BM adipocytes were physically located near melanoma cells in BM, we made a stable melanoma cell line labeled with GFP signal. GFP+ B16 cells were tracked in BM on days 6-7 post-tumor transplantation (Figure 4a). Immunohistochemistry staining of FABP4 was also performed to mark BM adipocytes in the sequential BM sections (Figure 4a). As shown in Figure 4a, adipocytes were in the vicinity of GFP+ melanoma cells in BM. The BM adipocytes became fibrotic, which was confirmed by Masson's trichrome staining, and were present in the tumor stroma (Figure 4b). These data support the hypothesis that BM adipocytes might influence the colonization of tumor cells in BM.

\section{BM Adipocytes Promote Melanoma Cell Proliferation and Cell Cycle Transitions}

To determine whether increased BM adipogenesis influences melanoma cell proliferation and cell cycle transitions in vitro, we exposed B16-F10 cells to conditioned medium derived from mature BM adipocytes (Figure 4c). Treatment with Ad-CM robustly increased B16-F10 cell proliferation compared with proliferation in the control group (Figure $4 \mathrm{~d}$ ). The G0/1 phases were decreased while the $S$ phase was significant upregulated in melanoma cells after Ad-CM exposure in a dose-dependent manner (Figure 4e). To confirm these phenotypes, we detected a panel of marker genes for cell proliferation and cell cycle transitions. As shown in Figure 4f, 
a

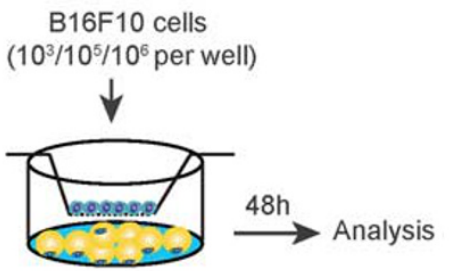

Mature BM adipocyte

(14F1.1) b

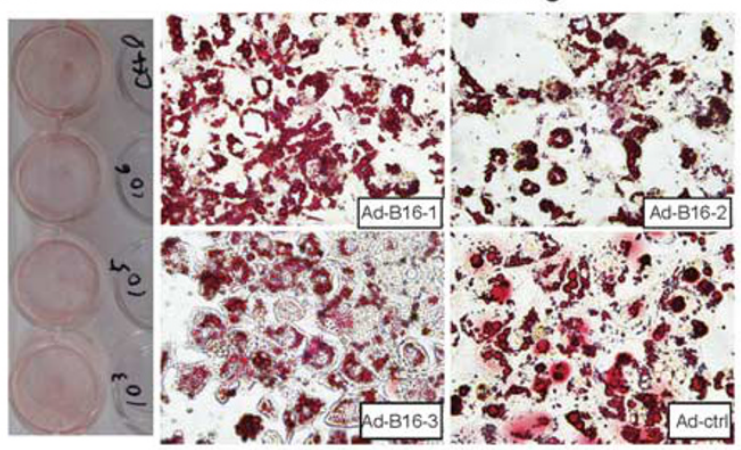

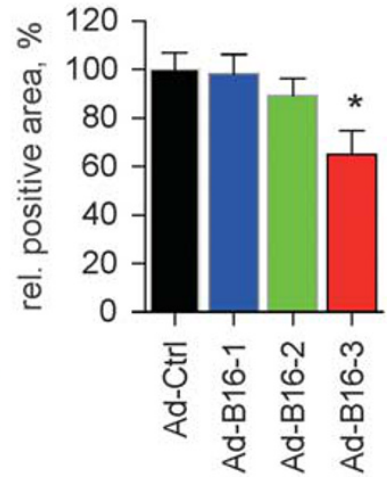

C Ad-ctrl Ad-B16-1

\section{Ad-B16-2}

Ad-B16-3

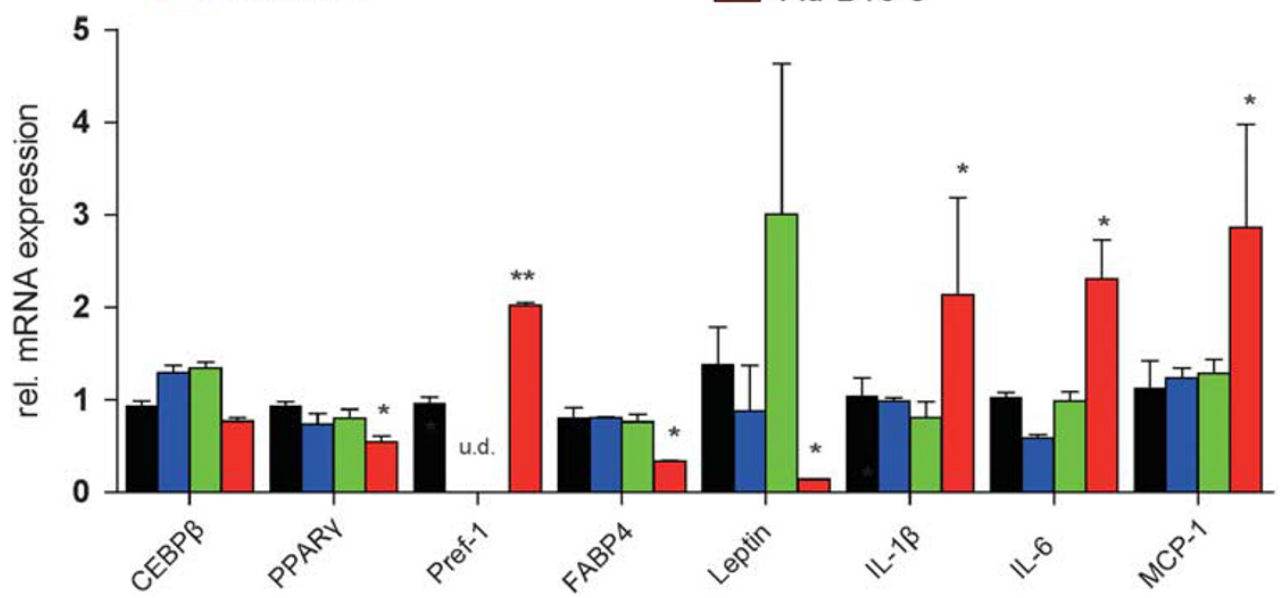

Figure 3 Tumor burden-dependent effects on BM adipocytes.(a) Scheme overview: 14F1.1 cells were coated on 24-well plates and differentiated into mature adipocytes and then co-cultured with $1 \times 10^{3}$ (Ad-B16-1), $1 \times 10^{5}$ (Ad-B16-2) or $1 \times 10^{6}$ (Ad-B16-3) B16F10 cells for an additional 48 h. BM adipocytes alone served as the control group (Ad-ctrl). (b) Representative pictures of Oil Red O staining of adipocyte cultures in 24-well plates. A representative image of at least three independent experiments carried out in triplicate is shown. Quantification of the intensity of staining was performed using ImageJ. (c) Gene expression in BM adipocytes from the above co-culture system. All of the data are the means \pm s.e.m. and are compared with the data from the Ad-ctrl group; three independent experiments were carried out in triplicate. u.d. means undetected, $* P<0.05$, ${ }^{*} P<0.01,{ }^{* * *} P<0.001$. BM, bone marrow.

the addition of Ad-CM to B16F10 cells resulted in increased mRNA levels of Cyclin Dependent Kinase 2 (CDK2) and Cyclin D1 and decreased mRNA levels of B-Cell CLL/Lymphoma 2 $(B C L-2)$ and $p 21$. Together, these results indicate that BM adipocytes facilitate melanoma cell growth in BM.

\section{BM Adipocytes Might Play a Role in the Alteration of the Tumor BM Microenvironment}

Melanoma bone metastases are commonly osteolytic metastases as a result of the aberrant activation of bone-absorbing osteoclasts. ${ }^{25,26}$ Therefore, a histomorphometric analysis was performed of the TRAP activity-stained tibial marrow sections. As shown in Figures $5 \mathrm{a}$ and $\mathrm{b}$, the largest differences in osteoclast size and number between cell-injected and vehicle-injected mice were observed on days 6 and 14 posttumor cell inoculation. Consistent with these results, trabecular bone volume and thickness were inversely correlated with osteoclast number and size on days 6 and 14 post-melanoma injection (Figures $5 \mathrm{c}$ and d). Noticeably, the numbers and sizes of the osteoclasts or trabecular bone volume were transiently restored to levels comparable to those in the PBS-injected mice at day 7 or 10 post-melanoma injection (Figures $1 \mathrm{~b}$ and $5 \mathrm{a}-\mathrm{d}$ ), indicating that the BM niche transiently recovered from the osteolytic BM microenvironment. Indeed, the time point for the increased $\mathrm{BM}$ adipogenesis was consistent with the alteration of the osteolytic BM microenvironment (Figures 1b-e). Furthermore, significant increases were observed in the serum levels of leptin (Figure 1e), which is functional in promoting osteoblast differentiation and proliferation and in suppressing osteoclastogenesis. ${ }^{27}$ Collectively, these data suggest that the increase in the number of BM adipocytes is associated with the modification of the osteolytic metastatic niche. 


\section{DISCUSSION}

In this study, we report that increased numbers of BM adipocytes support the development of melanoma bone metastasis. Our data suggest that BM adipocytes play a critical role in this development by providing a niche for cancer cell colonization and growth in BM. Increasing our understanding of the impact of BM adipocytes on tumor cells with osteotropism might therefore contribute to the development a
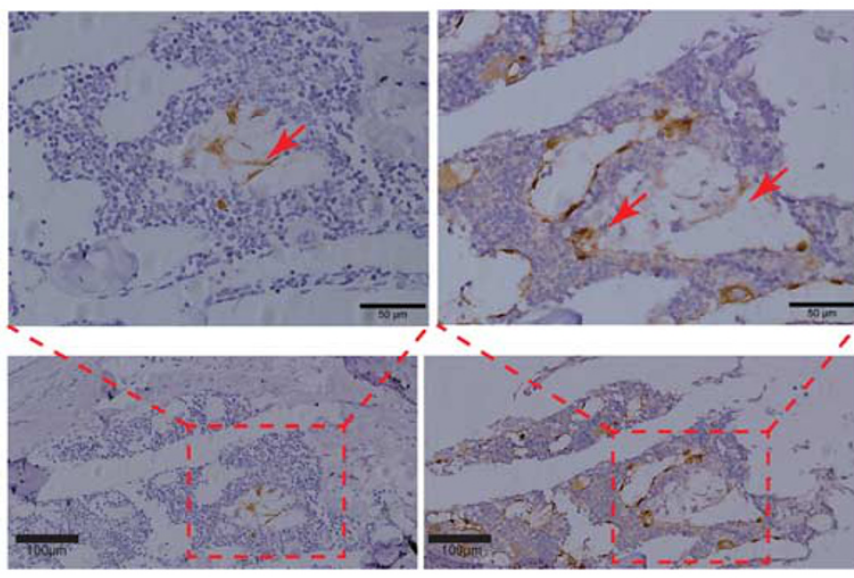

GFP

C

Adipocyte conditioned medium (Ad-CM) 0\%, 50\%, $100 \%$

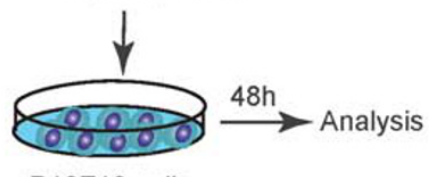

B16F10 cells

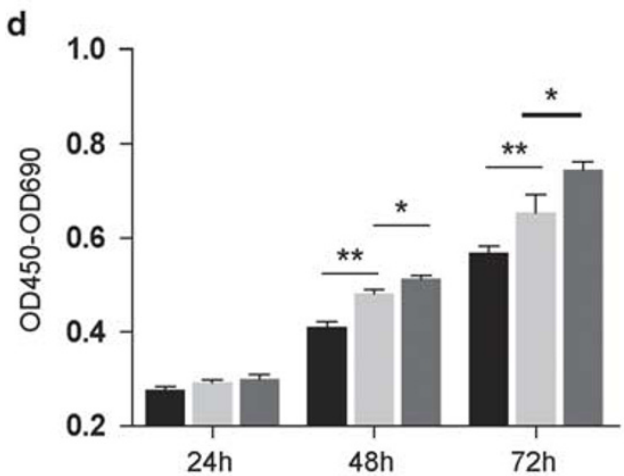

$f$

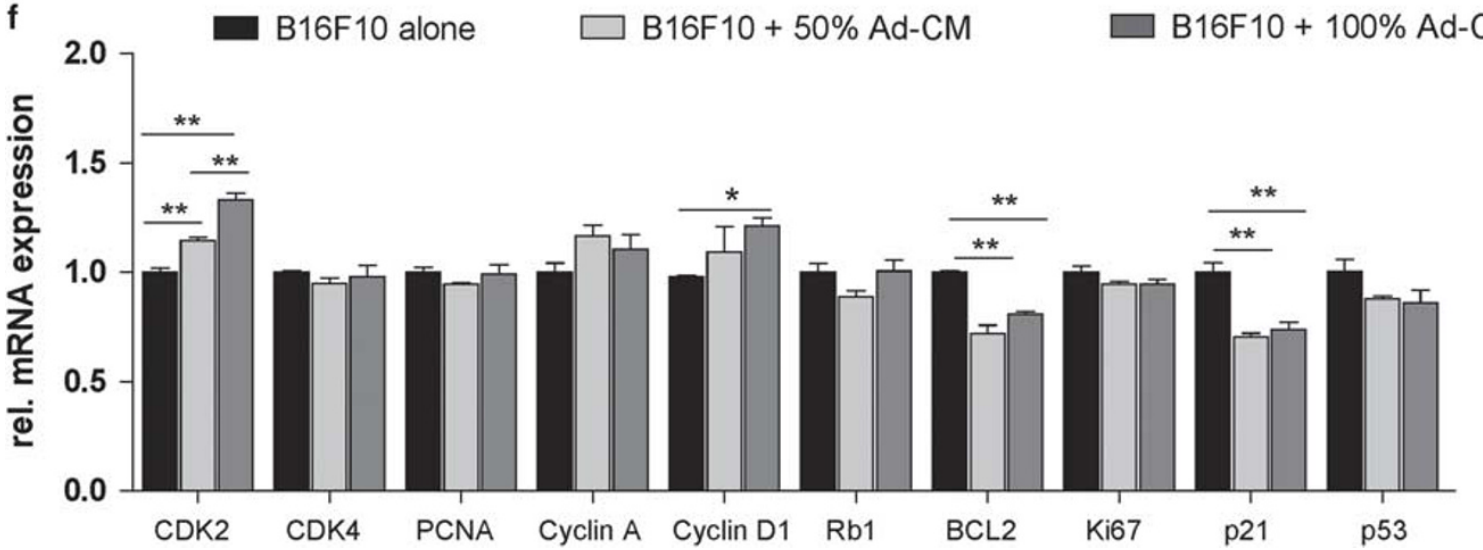

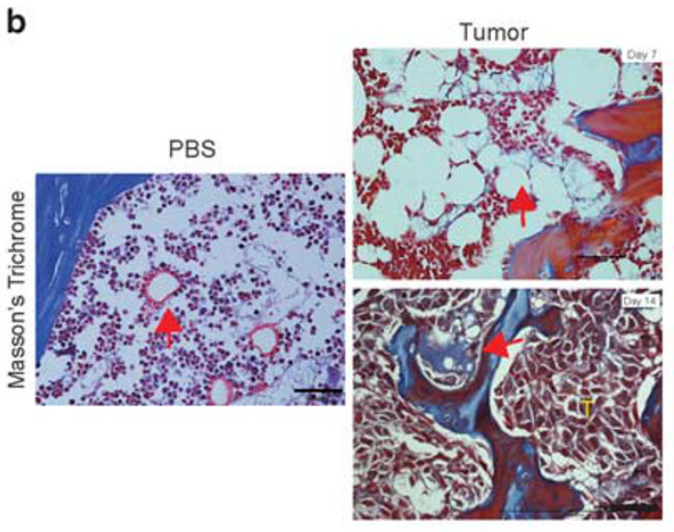

FABP4 e

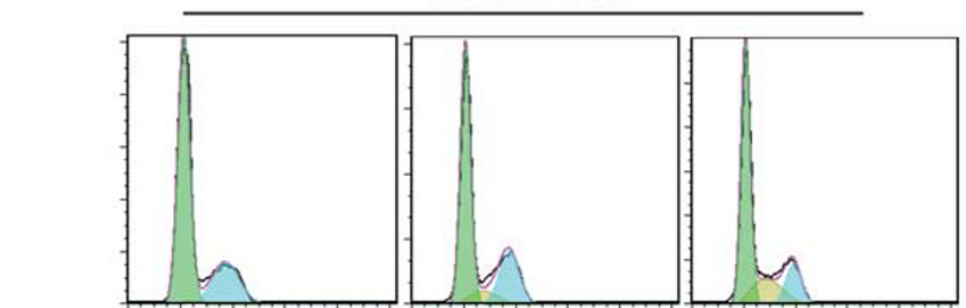

$50 \%$
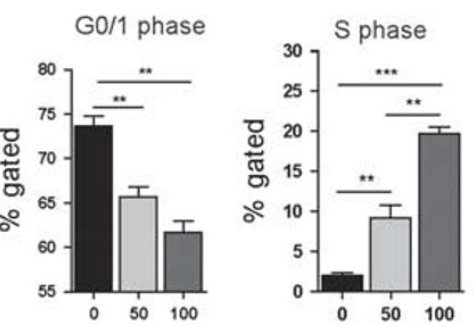
of novel therapeutic targets for the treatment of bone metastasis.

The mechanism by which the BM niche supports bone tropism in cancer cells remains largely unknown. In contrast to the osteogenic niche for metastasizing cancer cells, such as those observed in breast cancer ${ }^{6}$ and prostate cancer, ${ }^{4}$ we propose the presence of an adipogenic niche for cancer cell colonization and growth in BM in vivo. This concept is supported by a recent excellent in vitro study that was performed by Templeton et al. ${ }^{28}$ that showed that breast cancer cells can colonize the $\mathrm{BM}$ adipose tissue niche. It is also supported by evidence provided by Brown et al. ${ }^{29}$ showing that prostate cancer cells target arachidonic acid (AA)-loaded human BM adipocytes. In particular, obesity modeled by a high-fat diet ( $60 \mathrm{kcal} \%$ fat) in mice results in enhanced BM adipogenesis, ${ }^{16}$ and $\mathrm{BM}$ adipocytes occur in direct contact with cancer cells and promote tumor cell growth in BM..$^{11,12}$
Similarly, our findings from mice suggest that regardless of diet, BM adipocytes might also be essential for tumor cell colonization of bone metastasis.

In the present study, we observed that melanoma-derived factors were able to promote $\mathrm{BM}$ adipogenesis in vitro, which might partially account for the rapid increase in BM adipocyte number in the metastatic BM niche in vivo. Similarly, factors derived from leukemia, lung cancer cells, or ovarian cancer cells can enhance adipogenesis in vitro. ${ }^{23,30}$ It might be interesting to test whether BMP4 and IL-8 secreted by tumor cells can mediate the enhanced adipogenesis induced by melanoma cells and influence the process of bone metastasis. ${ }^{23}$ IL-8 influences the process of osteolytic bone metastasis in breast cancer. ${ }^{31}$ In addition, the differentiation of preadipocytes into adipocytes was found to be greater when the preadipocytes were in contact with breast cancer cell lines. ${ }^{32}$ Furthermore, Snail+ melanoma cells were observed to a
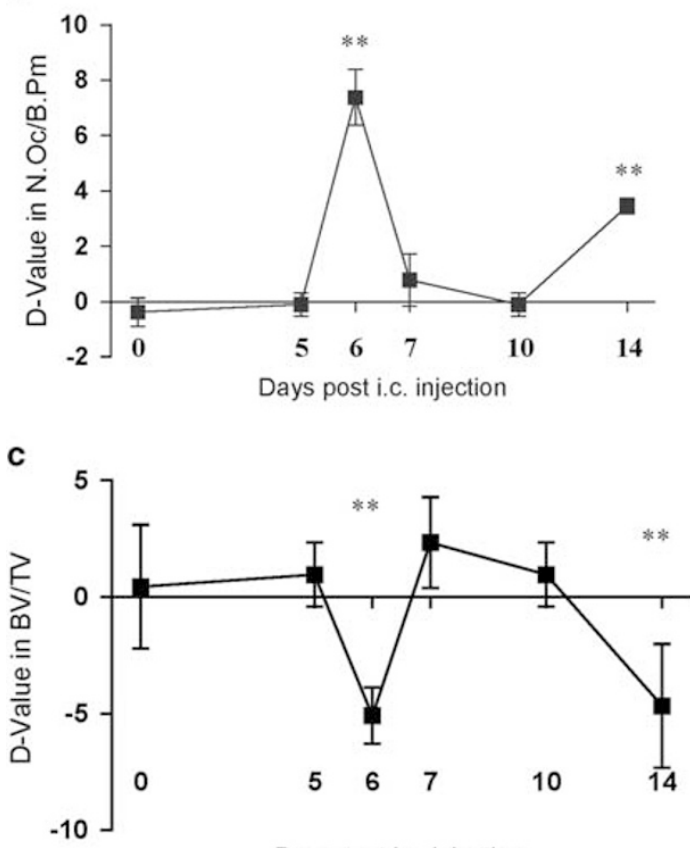

Days post i.c. injection b

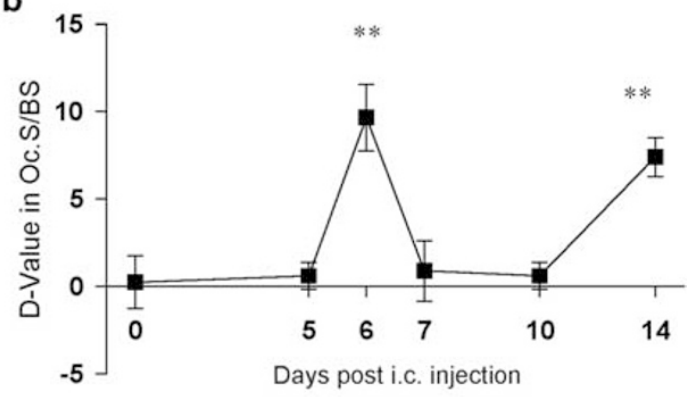

d

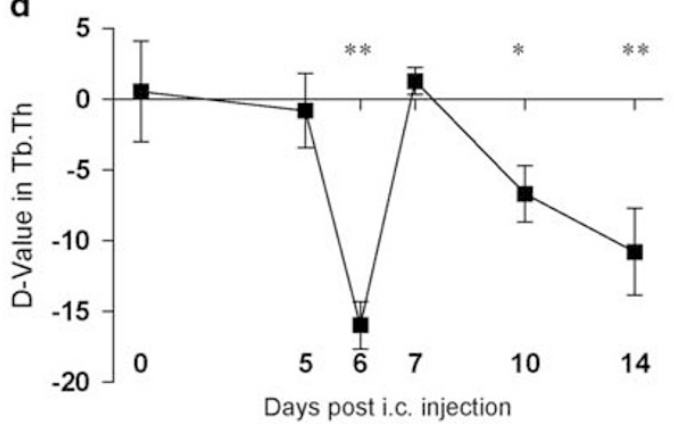

Figure 5 BM adipocytes might play a role in restoration of the osteolytic BM microenvironment. (a-d) Histomorphometric analysis of the proximal tibial metaphysis. The difference values (D-values) were calculated using the data from the tumor-injected mice and the vehicle-injected mice. N.Oc/B.Pm, osteoclast number/bone perimeter; BM, bone marrow; BV/TV, bone volume/total volume; Oc.S/BS, osteoclast surface/bone surface; Tb.Th, trabecular thickness. All of the statistical data are shown as means \pm s.e.m.; $n=5-6$ per group. ${ }^{*} P<0.05,{ }^{* *} P<0.01$.

Figure 4 BM adipocytes provide a niche for melanoma cell colonization and growth.(a) Representative photograph of GFP-labeled and FABP4-stained tibial marrow post day 7 intracardiac (i.c.) injection of B16-F10 cells. Scale bar, $50 \mu \mathrm{m}$. (b) Representative photographs of Masson's trichrome-stained tissues obtained on PBS, day7 and day 14 post i.c injection with B16-F10 cells. The red arrows indicate BM adipocytes. Tumor area is marked with a yellow ' $T$ '. Scale bar, $50 \mu \mathrm{m}$. (c) Experimental setting: B16F10 cells were incubated with medium mixed with different proportions of Ad-CM $(0 \%, 50 \%$, or $100 \%$ ) for 48 h. (d) WST-1 analysis of the cell proliferation in B16F10 cells treated with control or Ad-CM. The results of at least three independent experiments carried out in triplicate are shown. (e) Cell cycle was detected by flow cytometry and quantified after B16F10 cell treatment with a range of concentrations of Ad-CM for $48 \mathrm{~h}$. (f) Gene expression analysis of B16F10 cells incubated with Ad-CM. Three independent experiments were carried out in triplicate. ${ }^{*} P<0.05,{ }^{* *} P<0.01,{ }^{* *} P<0.001$. Ad-CM, adipocyte-conditioned media; $\mathrm{BM}$, bone marrow; $\mathrm{PBS}$, phosphate-buffered saline. 
generate a population of pluripotent mesenchymal stem-like CD45-ALCAM+ cells in the BM premetastatic niche via follistatin like 1 (FSTL1) that more frequently differentiated into mesenchymal lineages such as adipocytes and osteoblasts. ${ }^{33}$ These observations support the idea that the induced adipocytes might play a role in cancer development. However, the delipidation of BM adipocytes were also observed in vitro. The number of BM adipocytes diminished during tumor cell progression in vivo, suggesting a dual effect of tumor cells on BM adipocytes.

Our data suggest that the increased numbers of $\mathrm{BM}$ adipocytes might be involved in the transient restoration of the osteolytic BM microenvironment after melanoma cell colonization. However, BM adipocytes that promote osteoclast differentiation are well documented in the literature. ${ }^{13,22,27,34-36}$ Although there is controversy over the impacts of adipocytes on osteoblasts and osteoclasts, ${ }^{27}$ our observations suggest that BM adipocytes do not survive in a highly osteolytic BM microenvironment. Moreover, the peak in number of BM adipocytes occurred in a relatively intact $\mathrm{BM}$ microenvironment in terms of osteoclast size/number and trabecular bone volume.

In summary, our results support the concept that the enhancement of adipogenesis by invasive tumors ${ }^{23,30}$ may have a critical role in metastatic processes, including bone metastasis. BM adipocytes might play a pivotal role in bone metastasis by providing free fatty acids as an energy source for metastasizing cancer cell survival and growth. ${ }^{24,29,37,38}$ However, the lack of studies aimed at addressing BM adipocytes using lineage tracing or specific knockout mice precludes any definitive conclusions. The specific factors from cancer cells that are involved in inducing BM adipogenesis and its role in bone metastasis also require further investigation. Nevertheless, the observations of the present study are important because they provide insight into the cellular mechanisms underlying bone metastasis and support the notion that BM adipocytes are a potential therapeutic target for bone metastases.

Supplementary Information accompanies the paper on the Laboratory Investigation website (http://www.laboratoryinvestigation.org)

\section{ACKNOWLEDGMENTS}

This work was supported by grants from the Shanghai Science and Technology Committee, Shanghai International Communication Key Project (14430712000, 14430712001/14430712002). We thank Prof. Dov Zipori (Department of Molecular Cell Biology, The Weizmann Institute of Science, Rehovot, Israel), who kindly provided us with the murine bone marrow adipocyte line 14F1.1. We also thank American Journal of Experts (AJE) for English language editing. Grant numbers and sources of support: Shanghai Science and Technology Committee, Shanghai International Communication Key Project (14430712000, 14430712001/14430712002).

\section{DISCLOSURE/CONFLICT OF INTEREST}

The authors declare no conflict of interest.
1. Barth A, Wanek LA, Morton DL. Prognostic factors in 1,521 melanoma patients with distant metastases. J Am Coll Surg 1995;181:193-201.

2. Tas F. Metastatic behavior in melanoma: timing, pattern, survival, and influencing factors. J Oncol 2012;2012:647684.

3. Potepan P, Spagnoli I, Danesini GM, et al. The radiodiagnosis of bone metastases from melanoma. Radiol Med 1994;87:741-746.

4. Shiozawa $\mathrm{Y}$, Pedersen EA, Havens AM, et al. Human prostate cancer metastases target the hematopoietic stem cell niche to establish footholds in mouse bone marrow. J Clin Invest 2011;121:1298-1312.

5. Hanoun $M$, Zhang $D$, Mizoguchi $T$, et al. Acute myelogenous leukemiainduced sympathetic neuropathy promotes malignancy in an altered hematopoietic stem cell niche. Cell Stem Cell 2014;15:365-375.

6. Wang H, Yu C, Gao X, et al. The osteogenic niche promotes early-stage bone colonization of disseminated breast cancer cells. Cancer Cell 2015;27:193-210.

7. Krings $A$, Rahman $S$, Huang $S$, et al. Bone marrow fat has brown adipose tissue characteristics, which are attenuated with aging and diabetes. Bone 2012;50:546-552.

8. Justesen J, Stenderup K, Ebbesen EN, et al. Adipocyte tissue volume in bone marrow is increased with aging and in patients with osteoporosis. Biogerontology 2001;2:165-171.

9. Doucette $\mathrm{CR}$, Horowitz MC, Berry R, et al. A high fat diet increases bone marrow adipose tissue (MAT) but does not alter trabecular or cortical bone mass in C57BL/6 J mice. J Cell Physiol 2015;230:2032-2037.

10. Scutellari PN, Antinolfi G, Galeotti R, et al. Metastatic bone disease. Strategies for imaging. Minerva Med 2003;94:77-90.

11. Chen GL, Luo Y, Eriksson D, et al. High fat diet increases melanoma cell growth in the bone marrow by inducing osteopontin and interleukin 6 . Oncotarget 2016;24:26.

12. Herroon $\mathrm{MK}$, Rajagurubandara $\mathrm{E}$, Hardaway $\mathrm{AL}$, et al. Bone marrow adipocytes promote tumor growth in bone via FABP4-dependent mechanisms. Oncotarget 2013;4:2108-2123.

13. Hardaway AL, Herroon MK, Rajagurubandara $E$, et al. Marrow adipocyte-derived CXCL1 and CXCL2 contribute to osteolysis in metastatic prostate cancer. Clin Exp Metastasis 2015;32:353-368.

14. Nieman KM, Kenny HA, Penicka CV, et al. Adipocytes promote ovarian cancer metastasis and provide energy for rapid tumor growth. Nat Med 2011;17:1498-1503.

15. Naveiras $\mathrm{O}$, Nardi $\mathrm{V}$, Wenzel $\mathrm{PL}$, et al. Bone-marrow adipocytes as negative regulators of the haematopoietic microenvironment. Nature 2009;460:259-263.

16. Luo $\mathrm{Y}, \mathrm{Chen} \mathrm{GL}$, Hannemann $\mathrm{N}$, et al. Microbiota from obese mice regulate hematopoietic stem cell differentiation by altering the bone niche. Cell Metab 2015;22:886-894.

17. Schuettpelz LG, Link DC. Niche competition and cancer metastasis to bone. J Clin Invest 2011;121:1253-1255.

18. Bakewell SJ, Nestor P, Prasad S, et al. Platelet and osteoclast beta3 integrins are critical for bone metastasis. Proc Natl Acad Sci USA 2003;100:14205-14210.

19. Campbell JP, Merkel AR, Masood-Campbell SK, et al. Models of bone metastasis. J Vis Exp 2012;4:e4260.

20. Soleimani $M$, Nadri S. A protocol for isolation and culture of mesenchymal stem cells from mouse bone marrow. Nat Protoc 2009;4: 102-106.

21. Laharrague $\mathrm{P}$, Larrouy $\mathrm{D}$, Fontanilles $\mathrm{AM}$, et al. High expression of leptin by human bone marrow adipocytes in primary culture. FASEB $J$ 1998;12:747-752.

22. Holt V, Caplan Al, Haynesworth SE. Identification of a subpopulation of marrow MSC-derived medullary adipocytes that express osteoclastregulating molecules: marrow adipocytes express osteoclast mediators. PLoS One 2014;9:e108920.

23. Wong EWP, Shen L, Shieh J-H, et al. Enhanced adipogenesis of bone marrow-derived stromal cells by ovarian cancer cells via BMP4 and IL8 is associated with increase in cancer stem/progenitor cells and cancer cell proliferation. Cancer Res 2014;74:4866.

24. Dirat B, Bochet L, Dabek M, et al. Cancer-associated adipocytes exhibit an activated phenotype and contribute to breast cancer invasion. Cancer Res 2011;71:2455-2465.

25. Brountzos E, Panagiotou I, Bafaloukos D, et al. Bone metastases from malignant melanoma: a retrospective review and analysis of 28 cases. Radiol Oncol 2001;35:209-214.

26. Lau YS, Sabokbar A, Giele $\mathrm{H}$, et al. Malignant melanoma and bone resorption. Br J Cancer 2006;94:1496-1503. 
27. Muruganandan S, Sinal CJ. The impact of bone marrow adipocytes on osteoblast and osteoclast differentiation. IUBMB Life 2014;66:147-155.

28. Templeton ZS, Lie WR, Wang W, et al. Breast cancer cell colonization of the human bone marrow adipose tissue niche. Neoplasia 2015;17: 849-861.

29. Brown MD, Hart C, Gazi E, et al. Influence of omega-6 PUFA arachidonic acid and bone marrow adipocytes on metastatic spread from prostate cancer. Br J Cancer 2010;102:403-413.

30. Hirano $\mathrm{T}$, Morii $\mathrm{H}$, Nakazawa $\mathrm{K}$, et al. Enhancement of adipogenesis induction by conditioned media obtained from cancer cells. Cancer Lett 2008;268:286-294.

31. Bendre MS, Montague DC, Peery T, et al. Interleukin-8 stimulation of osteoclastogenesis and bone resorption is a mechanism for the increased osteolysis of metastatic bone disease. Bone 2003;33:28-37.

32. Delort $L$, Lequeux $C$, Dubois $V$, et al. Reciprocal interactions between breast tumor and its adipose microenvironment based on a 3D adipose equivalent model. PLoS One 2013;8:e66284.
33. Kudo-Saito C, Fuwa T, Murakami K, et al. Targeting FSTL1 prevents tumor bone metastasis and consequent immune dysfunction. Cancer Res 2013;73:6185-6193.

34. Goto $H$, Hozumi $A$, Osaki $M$, et al. Primary human bone marrow adipocytes support TNF-alpha-induced osteoclast differentiation and function through RANKL expression. Cytokine 2011;56:662-668.

35. Goto H, Osaki M, Fukushima T, et al. Human bone marrow adipocytes support dexamethasone-induced osteoclast differentiation and function through RANKL expression. Biomed Res 2011;32:37-44.

36. Takeshita $S$, Fumoto $T$, Naoe $Y$, et al. Age-related marrow adipogenesis is linked to increased expression of RANKL. J Biol Chem 2014;289: 16699-16710.

37. Bochet L, Lehuede C, Dauvillier S, et al. Adipocyte-derived fibroblasts promote tumor progression and contribute to the desmoplastic reaction in breast cancer. Cancer Res 2013;73:5657-5668.

38. Morris EV, Edwards CM. The role of bone marrow adipocytes in bone metastasis. J Bone Oncol 2016;5:121-123. 